\title{
e-Phaïstos
}

e-Phaïstos

Revue d'histoire des techniques / Journal of the history

of technology

III-1 | 2014

Varia

\section{L'activité du koudougou ou de la forge à Sadaba (province de l'Oubritenga, Burkina Faso) : la transmission d'une technique entre méthodes anciennes et innovations}

The activity of koudougou or mine in Sadaba (Oubritenga Province, Burkina

Faso): the transmission of a technique between old methods and innovations

\section{Élise-Foniyama Thiombiano-Ilboudo}

\section{OpenEdition}

Journals

Édition électronique

URL : https://journals.openedition.org/ephaistos/581

DOI : 10.4000/ephaistos.581

ISSN : 2552-0741

Éditeur

IHMC - Institut d'histoire moderne et contemporaine (UMR 8066)

Édition imprimée

Date de publication : 1 juin 2014

Pagination : $59-72$

ISSN : 2262-7340

Référence électronique

Élise-Foniyama Thiombiano-Ilboudo, «L'activité du koudougou ou de la forge à Sadaba (province de I'Oubritenga, Burkina Faso) : la transmission d'une technique entre méthodes anciennes et innovations », e-Phaïstos [En ligne], III-1 | 2014, mis en ligne le 17 novembre 2016, consulté le 28 juin 2022. URL : http://journals.openedition.org/ephaistos/581 ; DOI : https://doi.org/10.4000/ephaistos. 581 


\title{
L'activité du koudougou ou de la forge à Sadaba (province de l'Oubritenga, Burkina Faso) : la transmission d'une technique entre méthodes anciennes et innovations
}

\author{
Docteur THIOMBIANO-ILBOUDO \\ Elise Foniyama \\ Maitre assistant, UFR/SH, département \\ d'Histoire et d'Archéologie \\ Université de Ouagadougou, Burkina Faso
}

Le village de Sadaba se situe à 7 kilomètres du village de Yamané dont il dépend et à 77 kilomètres environ au nord-est de la capitale Ouagadougou. L'activité de koudougou est une activité ancestrale et exécutée depuis un siècle par une même famille dans ce village. La forge est un lieu de travail avec une organisation qui lui est propre. Elle constitue également le centre de formation des jeunes. Pour comprendre les aspects techniques et les modes de transmission dans une société dominée par l'oralité, nous allons structurer notre travail en trois parties. La première portera sur l'origine et les fonctions des forgerons, la seconde sur le koudougou ou forge, son organisation et les différentes techniques de production, et la troisième sur les procédés de transmission des savoirs.

\section{L'origine et les fonctions des forgerons de Sadaba}

L'origine des forgerons
Les forgerons de Sadaba ont pratiqué traditionnellement le métier de métallurgistesforgerons car ils assuraient la chaîne opératoire de la production du métal mais aussi celle des outils finis. Chez les Bassar de l'Ouest du Togo, Philippe de Barros ${ }^{1}$ distingue une "smelting zone " et une «smithing zone» 2 . Cependant, Stéphan Dugast observe la présence simultanée des pratiques de fonte et de forge à la frontière de ces deux zones3. Chez les Moose de Sadaba, il n'y a pas de division du travail comme le constate Georges Celis qui indique que "Dans la société à trois composantes du Burundi et du Rwanda, on constate que les Tutsi ne forgent jamais tandis que les Twas (Pygmoïdes) forgent rarement, leur compétence se limitant à la fabrication d'armes et d'outils. Les forgerons et les fondeurs sont des Hutus »4. À Sadaba, les forgerons occupent deux quartiers à savoir Pïngin et Tenkeingseega. À leur arrivée à Sadaba, les forgerons les plus proches étaient ceux du village de Razoogtenga, situé à 3 kilomètres à l'ouest. Originaires de Yelkoto qui serait leur point de 


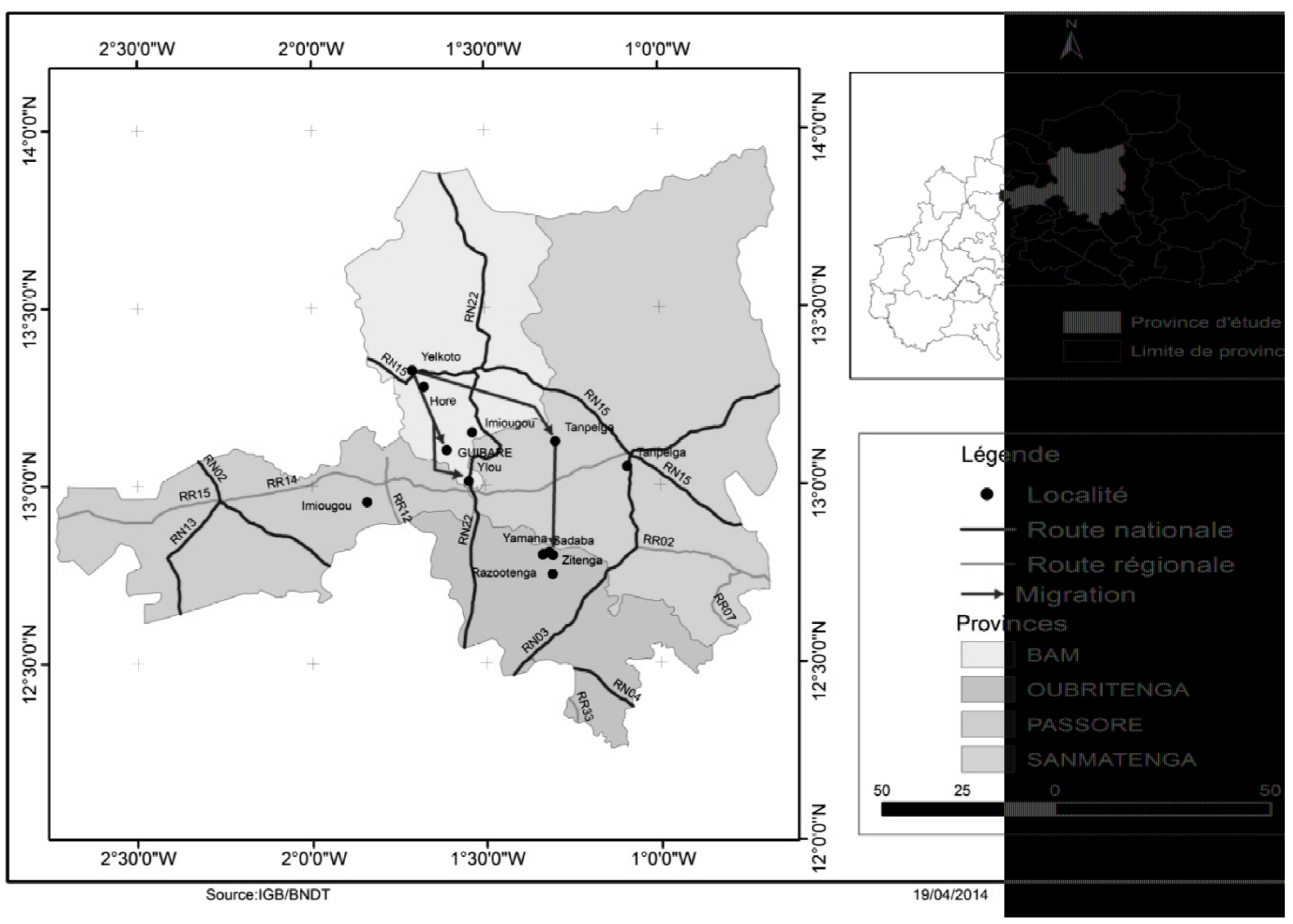

Figure 1 : Carte de localisation

départ, les forgerons de Sadaba se sont installés à Horé5, il y a plus de 130 ans. De là il y eut une dispersion vers Yilou, Guibaré, Tanpelga (à côté de Zitenga, chef-lieu de département). Ceux de Sadaba viendraient de Tanpelga (cf. fig. 1). Ils se sont installés à Sadaba il y a au moins 110 ans 6 . Depuis leur plus jeune âge, des métallurgistes ont participé à la réduction du minerai de fer7. Leur nom de famille « Nikiéma » est une déformation du mot en langue mooré "niin cegma» qui signifie «personne de bonne condition physique ». Ce nom leur a été attribué en référence au métier pénible de métallurgiste. Autrefois, ils ne nouaient pas de relations matrimoniales en-dehors du clan des métallurgistes, mais de nos jours, leurs femmes ont des origines diverses.

Ils livraient leurs productions aux villages de Sadaba, Zitenga, Imiougou et Yamana. Les sãnba (forgerons) de Sadaba, Tanpelga, Yilou, Guibaré, Horé et Yekoto sont de la même famille et le sannaaba (chef forgeron) de Sadaba est le plus vieux de toutes ces familles qui lui doivent allégeance. La forge appartient à la communauté et son chef est le plus vieux du lignage. 
Les autres forgerons moose que l'on trouve aujourd'hui dans les provinces environnantes (Yatenga, Sanamatenga et Bam) portent le nom de Bamogo, Zorom, Kindo, Yampa, Gansonré et Nikiéma.

En plus du travail de forge, le forgeron exerce de nombreuses autres fonctions. À Sadaba, il est guérisseur, exorciste, médiateur, etc. Jean-Baptiste Kiéthéga indique à propos du Burkina Faso que «le forgeron peut cumuler plusieurs fonctions dont celles de circonciseur, exciseur (ou exciseuse pour les femmes), guérisseur, rebouteur, chirurgien, exorciste, gynécologue, avorteur, dentiste, croquemort, bourreau, juge, négociateur de paix et de mariage, conseiller, ministre, etc... » 8 .

$\grave{A}$ Sadaba, le forgeron est craint et parfois méprisé. Leur origine du nord du Burkina explique cette situation. Les métallurgistes suscitent la méfiance car la population leur attribue des pouvoirs mystiques. Par exemple, en cas de foudre, c'est à eux que l'on fait appel. La foudre est un élément naturel qui est très craint par les populations, qui pensent très souvent qu'elle est d'origine surnaturelle. Les forgerons par leur aptitude à dompter cet élément sont par conséquent craints. En effet, ils sont les seuls habilités à toucher le corps d'une personne foudroyée grâce à leur maîtrise de l'art du feu. Les forgerons interviennent par des rites pour extraire la "hache de foudre» avant la levée du corps. Pour ce qui est du mépris, la population trouve le travail de métallurgiste ou de forgeron salissant et non noble. C'est pourquoi, certaines ethnies ou groupes socioprofessionnels interdisent le mariage avec eux. Par exemple, une personne issue de la famille royale est considérée comme noble et ne peut en aucun cas se marier à un forgeron. Mais d'autres raisons guident aussi les relations matrimoniales. À cet effet, Jean-Baptiste Kiéthéga indique que «les forgerons du Moogo et de pays bisa9 ignorent l'endogamie. Par contre, ils ne nouent pas de relations matrimoniales avec les Benda $^{10}$, Fulbé ${ }^{11}$, Nakomsé ${ }^{12}$, Poésé ${ }^{13}$, Setba ${ }^{14}$ et Yarsé $15 »^{16}$. Les causes de l'évitement sont la recherche de la sécurité de la technique des forgerons. Cette attitude est inconnue chez les Gulmanceba (population qui occupe l'est du Burkina Faso) où le forgeron est intégré dans la société. En somme, au Burkina Faso, les comportements sont donc variables selon les communautés de métallurgistes. Mais quelle que soit l'origine et les pratiques matrimoniales des forgerons, pour mener à bien leur mission, l'espace de travail connaît une organisation bien élaborée.

\section{L'organisation sociale et spatiale de la forge}

\section{L'organisation spatiale}

Tout chef de famille peut implanter un atelier de forge à côté de sa maison, mais il reste lié à la famille mère où se trouve le san-naaba ${ }^{17}$. Dans la société moaaga, on distingue des forgerons de naissance "rognè-saado » et de conversion «saa$k i e ̂ d b a »^{18}$. À Sadaba, il n'est pas interdit aux autres membres du village désireux d'apprendre le métier de le faire, mais nous ne connaissons aucun membre étranger au groupe Nikiéma. L'organisation spatiale de la forge respecte un certain nombre d'interdits et de faits mystiques.

L'atelier de forge se situe en général dans le village, à côté des cases. L'aménagement est très simple, sous un hangar soutenu par quatre fourches en bois et couvert d'un toit de paille. On retrouve le même type d'aménagement chez les forgerons gulmanceba de Fada N'Gourma à l'est du Burkina Faso où le toit est recouvert de tiges de mil ${ }^{19}$. En Ouganda, «il [en] existe de toutes les sortes et de toutes les dimensions : cela va de la petite hutte hémisphérique de style ancien (dont le toit de chaume descend jusqu'à terre) au simple toit rectangulaire et plat soutenu par quelques piquets et sans parois latérales ». Le koudougou ou la forge moaaga de Sadaba est composée d'un ou de plusieurs foyers, yégré au singulier ou yèga au pluriel, d'un kĩncirga ou esprit de la forge, du 
collecteur $\left(\right.$ pebga $^{20}$ ) et des tuyères ${ }^{21}$ ou pebsé (cf. fig. 2).

Dans les ateliers de forge, aucune autre activité ne peut être menée. Cela contraste avec ce que l'on rencontre chez les Fikos du Mali où il est signalé que "The internal space is divided into areas for working metal and wood. The external space is also used for various purposes related to production $»^{22}$. cœur même du foyer. Celle située au nord supporte une construction latérale d'une hauteur d'environ $10 \mathrm{~cm}$ qui forme un arc. Cette construction sert à freiner le flux d'air. Elle permet d'éviter également que la pierre soit directement en contact avec la source thermique, ce qui conduirait à son éclatement ou à son effritement. Les deux autres pierres maintiennent le collecteur qui débouche dans le foyer.

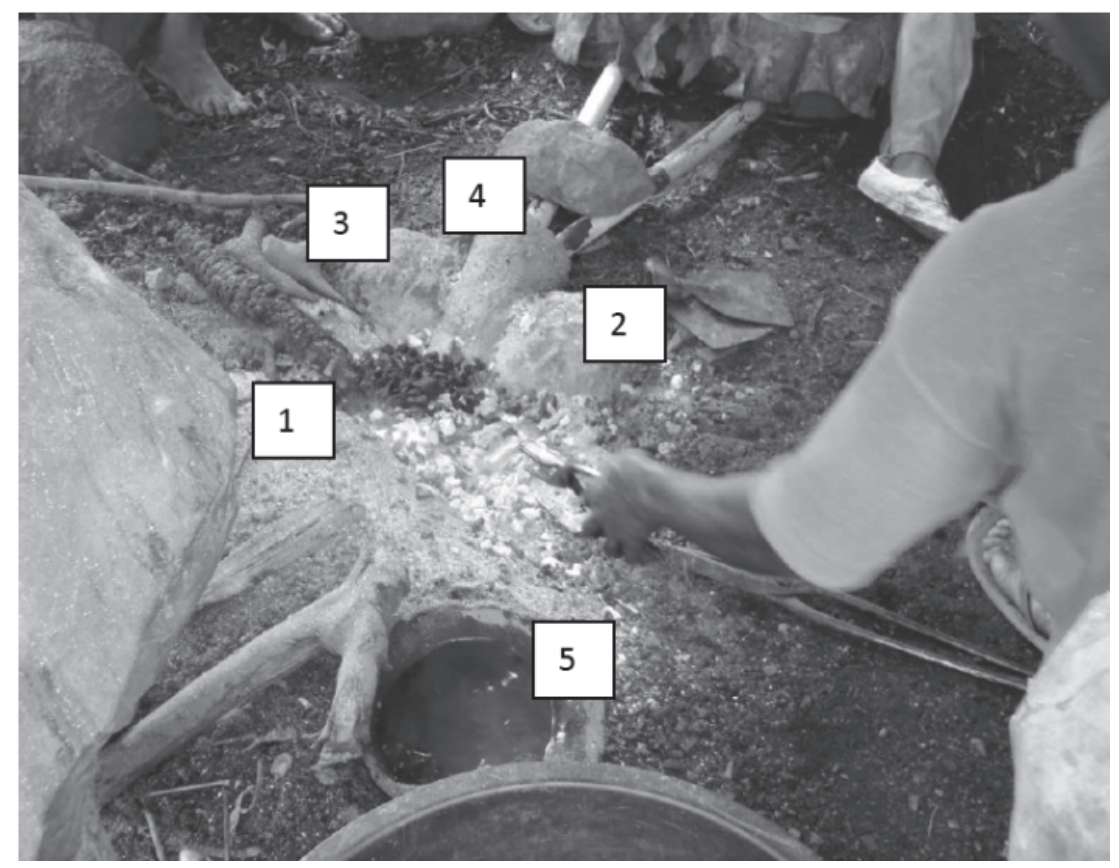

Fig. 2 : foyer de forge.

Légende :

1,2 et $3:$ yèga

4: pebga

5 : koudbaaré

Le foyer est constitué d'un simple creux qui contient du charbon de bois utilisé comme combustible. Chaque foyer est composé de quatre pierres, dont trois délimitent le foyer orienté vers l'est. La première est placée au nord, la seconde au sud-ouest et la troisième au sud-est. La quatrième pierre, dont la présence n'est connue que des forgerons, est enterrée au centre. Les trois pierres sont entièrement recouvertes d'argile. Elles protègent la flamme et constituent les limites du
Le collecteur et le kĩncirga sont orientés vers l'est. Le collecteur rassemble les tuyères qui envoient l'air dans le foyer. Le kĩncirga est l'esprit de la forge ou la divinité représentée sous forme d'une construction en argile. Le kĩncirga est coincé entre le collecteur et la pierre placée au sud-ouest du foyer. Autrefois, les deux tuyères en argile séchée étaient fixées à deux pots de terre et conduisaient l'air dans le collecteur, qui à son tour transférait l'air dans le foyer où il est implanté directement. De 
Figure3: équipe de forgerons

\author{
1 : Kougtaoré \\ 2 : Songda \\ 3: Zougda \\ 4:Zamsda $=$ apprenti
}

Légende :

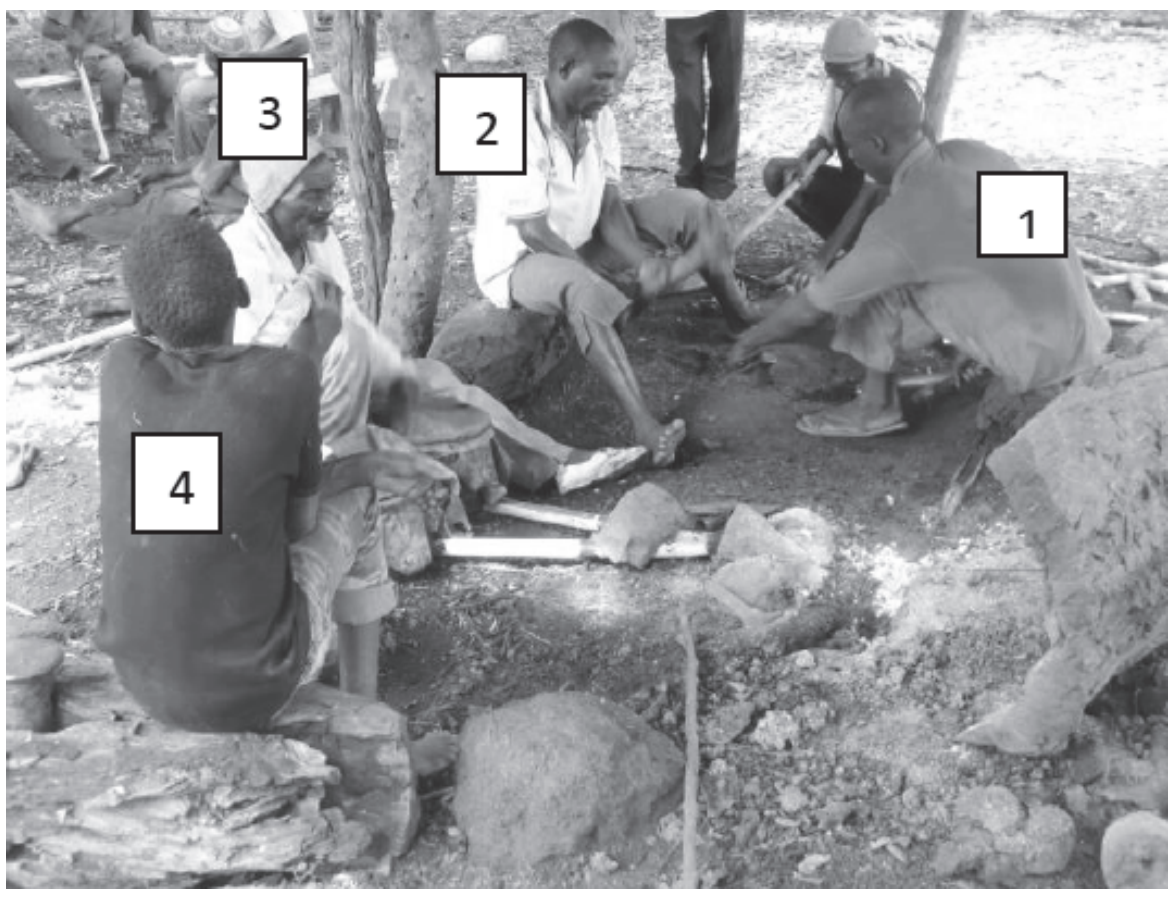

nos jours, les pots en argile et les tuyères sont parfois confectionnés à partir d'une matière plus résistante, notamment le fer. Quant au kĩncirga, il veille à la protection de la forge contre les accidents et les mauvais esprits, offre la fécondité aux femmes stériles de la société ou guérit les malades. Il est de temps à autre nourri du sang de poulets qu'on lui sacrifie. Ce sang est aspergé sur les différents objets de l'atelier de forge. Si telle est l'organisation de l'espace de la forge, un personnel spécialisé permet de la mettre à profit pour réaliser le travail.

\section{L'organisation sociale de la forge}

Dans un atelier de forge, il est nécessaire de bénéficier du travail d'un certain nombre de personnes aux rôles bien définis. Les quatre points cardinaux ont une signification dans l'organisation $\mathrm{du}$ foyer mais aussi dans la disposition du personnel.
Le kougtaoré, placé au nord de l'atelier, est assis dos tourné vers l'est. C'est le forgeron, maître du jour car il dirige les travaux du foyer. C'est lui qui retire l'objet du feu lorsqu'il estime qu'il est assez chauffé, guide le martelage, la trempe, donne le signal pour le lissage et toutes les actions de la forge. Placé à un mètre au nord-ouest, il fait face à un aide qui martèle le fer avec lui.

Le kougtaoré est positionné obliquement par rapport au zougda (souffleur) qui se situe à l'est du foyer (cf. fig. 3).

Le koudpooré se localise au nord, c'est l'arrière de la forge. Cet espace englobe le dépotoir de la forge localisé aussi à l'ouest, constituant ainsi un arc de cercle. Un pare-vent en bois se trouve à l'est à moins d'un mètre du foyer. Il freine le courant d'air vers le foyer, qui souffle d'est en ouest. Le manque de protection du foyer, dû au fait qu'il est exposé au vent de tous les côtés, conduit à cette mesure de pare-vent. Il est confectionné à partir d'un tronc 
d'arbre dont le tiers est coupé dans la longueur, puis l'intérieur décapé, donnant une forme arrondie à l'objet. Il possède environ cinq centimètres d'épaisseur et présente la forme d'une demi-lune allongée. L'accès à la forge peut se faire au niveau des quatre points cardinaux. Dans sa simplicité, la forge des Moose de Sadaba ressemble à celle décrite au Burundi à quelques exceptions près. C'est ainsi que Georges Celis dit : «Par contre, sur le plan des techniques, les ateliers de forge sont d'une parfaite uniformité ; le foyer (izï̌ko, le feu) est un simple creux dans le sol, il fonctionne au charbon de bois (amakára) et est activé par un soufflet double en bois (umu-vuba) régionalement, le soufflet en bois est remplacé parfois par une paire de soufflets simples en argile séchée (inkúunga) ; une tuyère en argile séchée (inkéro) conduit l'air du soufflet au foyer ».23

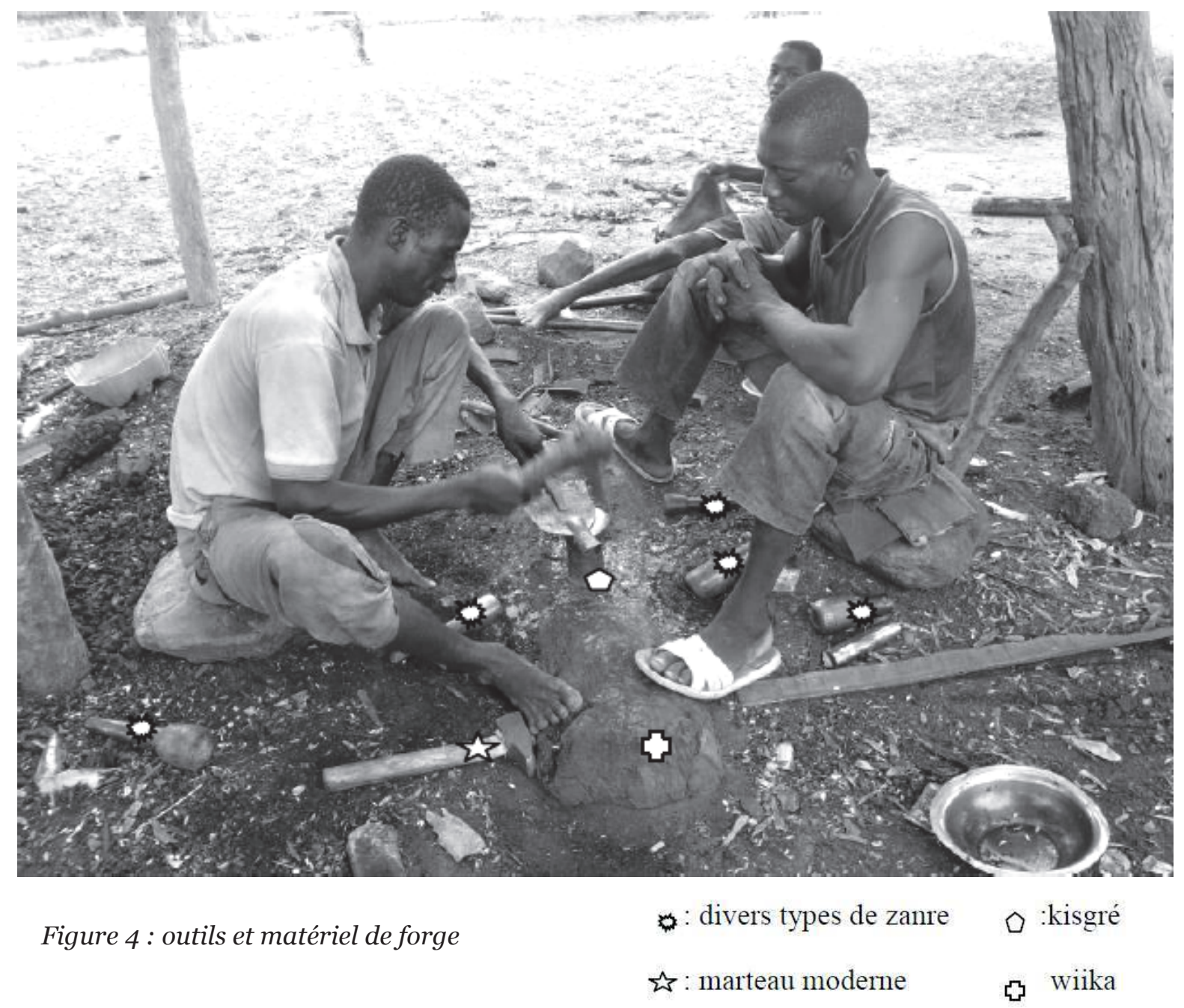




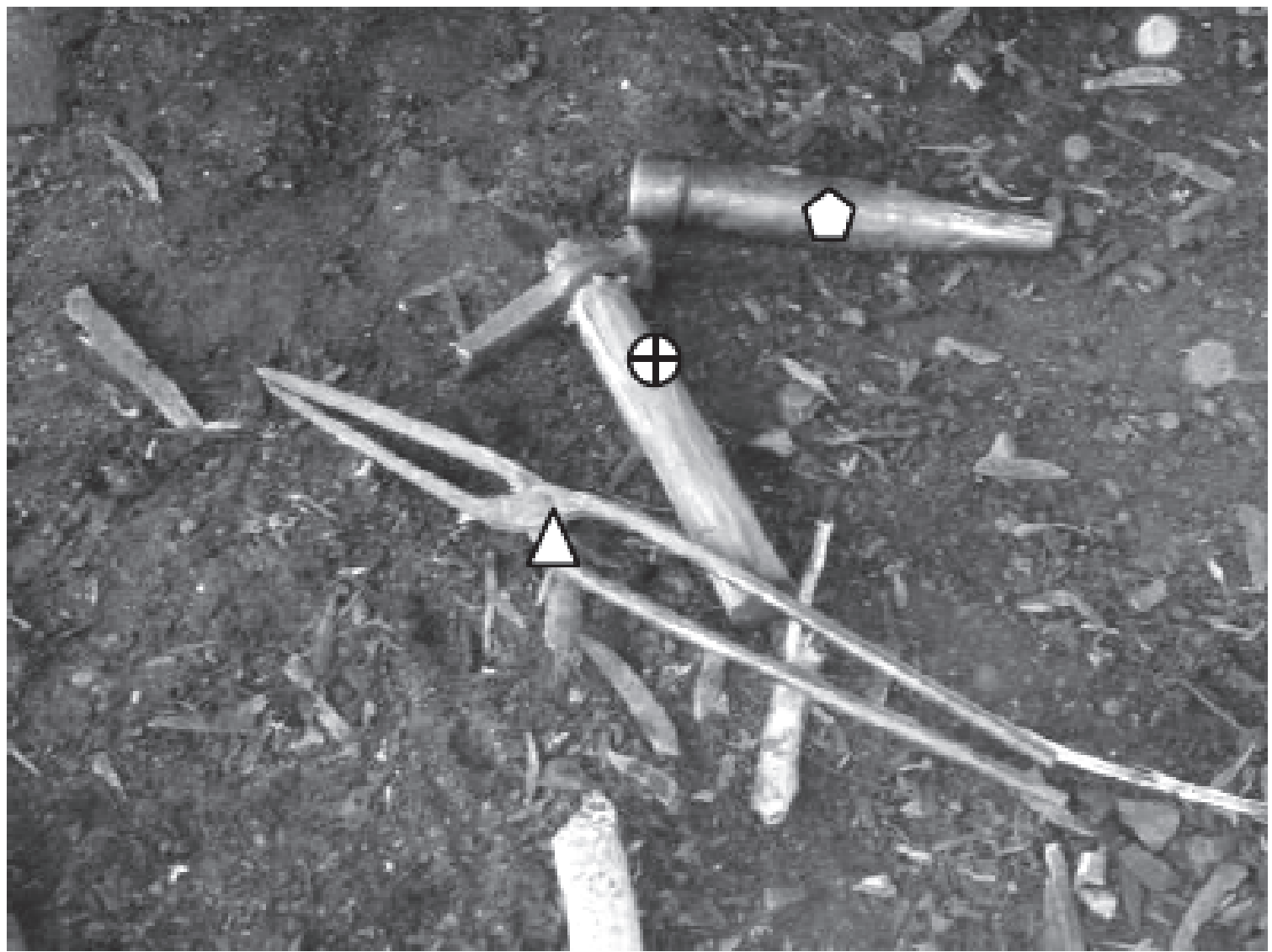

Figure 5 : Outils de forge

Les outils de la forge sont nombreux (cf. fig. 4 et 5). On compte parmi ces objets le zãnré ou marteau. On en distingue un petit appelé le zãnbila et un grand dit zãnbedré. Le zãnré est aussi un objet de médiation qui symbolise la puissance et la gloire. Il joue également le rôle de messager dans le cas de l'annonce des nouvelles difficiles, notamment les décès. Le koadga, qui une sorte de marteau, se divise lui aussi en koadbila (encore appelé wirgo, il $\triangle$ : yigo $\oplus:$ marteau moderne

$\triangle: k o a d b e d r$-tasbo sert à donner une forme par une technique de torsion) et koadbedr-tasbo (un type de marteau dont le percuteur est un peu plus gros que le reste du corps). Il a pour rôle d'amincir l'objet par un martelage qui le fait glisser sur l'objet. Il est destiné au finissage des objets. Le wiika est le support en bois dans lequel on place le kiisgré ou enclume. Il joue un rôle très important car il empêche l'enclume de s'enfoncer à cause de la pression due au 


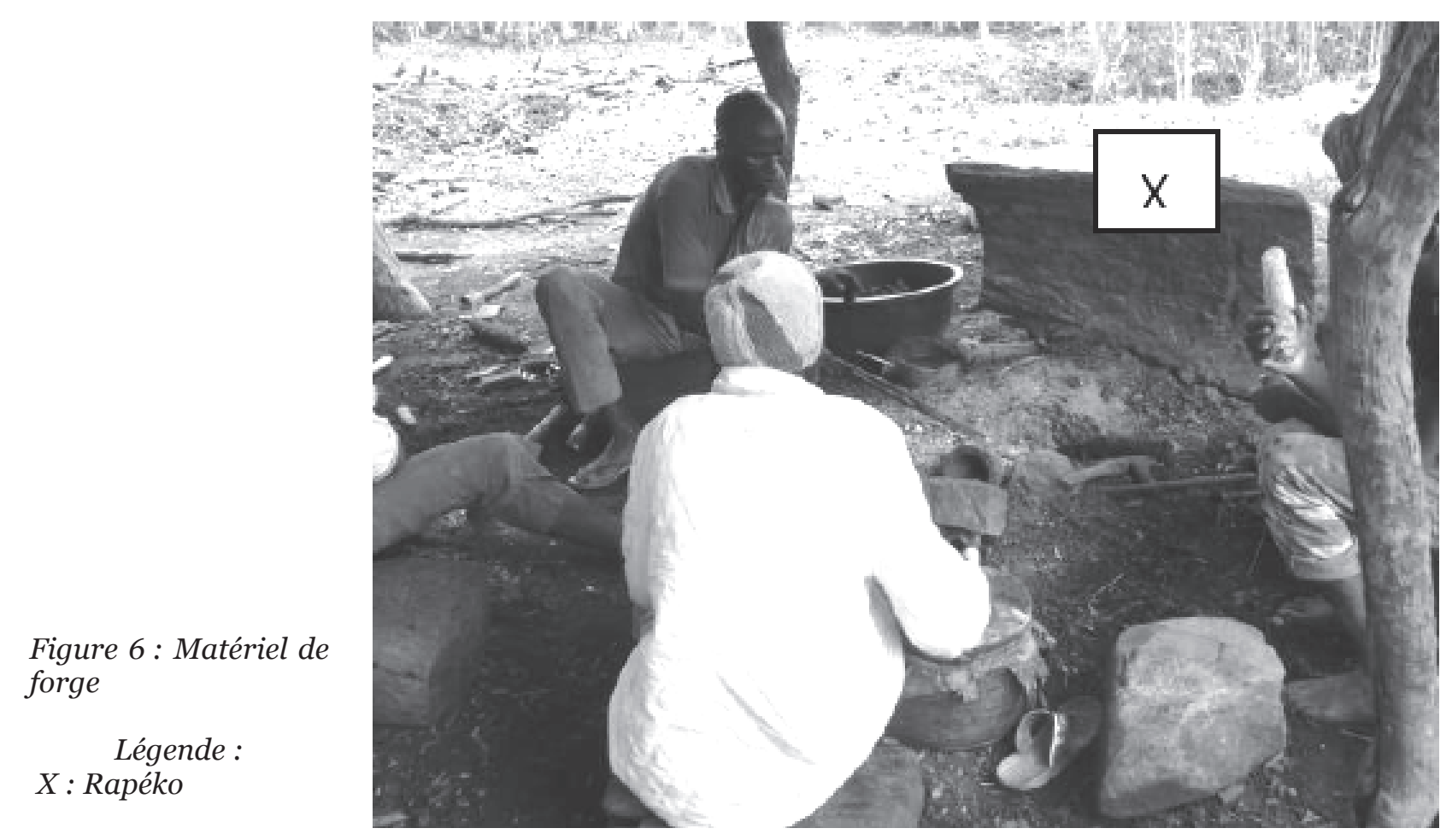

martelage. Ainsi, le bois a la propriété de retenir l'enclume dont la pointe fixée directement au sol a tendance à disparaître dans celui-ci après une longue période de martelage. L’espèce végétale utilisée pour sa confection est le caillcédrat (Kaya senegalensis) ou le karité (Butyrosnum parkii) choisis par les forgerons qui les considèrent comme des espèces très résistantes. Orienté nord-sud, il mesure environ $2 \mathrm{~m}$ de long et $1 \mathrm{~m}$ de large. Pendant le martelage, les deux forgerons qui se font face prennent leur appui en posant leurs pieds contre le wiika. On trouve également dans la forge le yigo ou pince qui sert à retirer les objets du feu.

Il y a également le kiisgré ou koudgou (enclume) qui mesure en moyenne $25 \mathrm{~cm}$ avec un diamètre de $10 \mathrm{~cm}$. La partie haute est plus large que la partie basse destinée à s'enfoncer dans le sol ou dans un bois. Elle révèle une forme de cône dont la pointe mesure en moyenne $3 \mathrm{~cm}$. L'enclume était confectionnée à partir de la loupe de fer, mais de nos jours la rareté de la loupe a conduit à la fabriquer à partir de fer de récupération.

Le koudbaaré désigne le récipient contenant l'eau de refroidissement des objets.

Suite à une chauffe et à un martelage, l'objet subit la trempe qui lui donne un certain nombre de qualités qui contribuent à sa résistance. Par les procédés de coupe, de martelage, de soudure, de torsion et de trempe, les forgerons fabriquent les objets suivants : laaré (hache), koutoaga (lame de daba servant à semer), soanga (lame de petite daba), fouguoonga (aiguille), sagg gnaggna (barrette traditionnelle à cheveux), gelbré (aiguille pour cheveux), baarga (lame de circoncision), bougtanga (fer servant à faire du feu par percussion 
d'une roche ou allumette traditionnelle), salbré (mors de cheval), souuga (couteau).

Les objets étaient écoulés par troc, par don et par un engagement ou sardo (c'est un contrat par lequel une personne s'engage à donner une compensation en nature (exemple le mil) à la fin des récoltes au forgeron). Pour que le métier se perpétue, la formation est assurée à la forge par des procédés spécifiques.

\section{Les procédés de transmission des savoirs}

La forge est une école, un lieu de fabrication des outils mais surtout de formation des jeunes pour la pérennisation de l'activité affirme Salif Nikiéma ${ }^{24}$. Les mécanismes de transmission des savoirs et savoir-faire sont liés au type de société. Ici, il s'agit d'une population où la culture de l'oralité occupe encore une place très importante malgré le développement actuel de l'écriture. Aussi, les dispositifs de transmission dans ce milieu sont

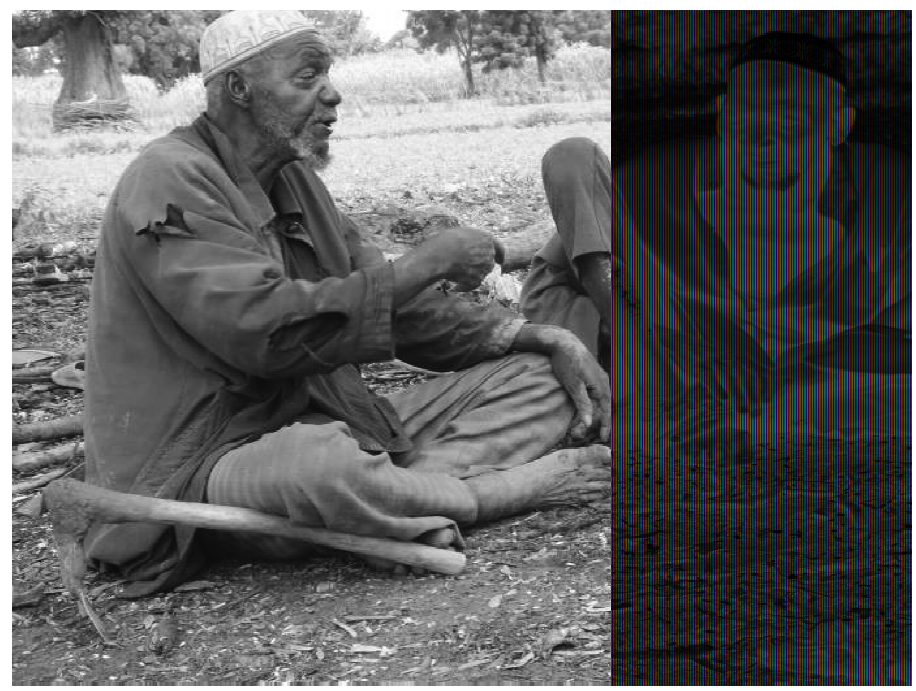

Figure 7: Recueil de tradition orale auprès de vieux métallurgistes l'admission de l'enfant en bas âge à la forge, sa participation à la soufflerie, l'attention et la pratique. L'apprentissage se fait très tôt chez les forgerons moose de Sadaba, nous confient les vieux métallurgistes (cf. fig. 7).

À propos des forgerons moose du Yatenga au nord du Burkina Faso, Martinelli indique que «Le jeune enfant est présent à toutes les activités qui se déroulent à la forge familiale dès son jeune âge. On dit ainsi qu'il naît dans la forge $»^{25}$. La formation est volontaire et sans distinction de sexe. Avant l'âge de cinq ans déjà l'enfant fréquente la forge où travaillent les membres de la famille. Dès qu'il en est capable, il peut actionner les soufflets, première étape de l'apprentissage. La durée d'initiation dépend des capacités d'assimilation de chaque enfant.

Les filles forgeronnes sont également associées au travail de la forge pour la soufflerie. Elles ne sont donc pas exclues de la forge comme il a été observé par Raphaëlle Soulignac et Vincent Serneels chez les Fiko du Mali, lesquels indiquent que «woman are excluded from the smithing workshop $»^{26}$. Chez les Moose de Sadaba, les filles forgeronnes participent traditionnellement à une seule étape de la fabrication des objets, à savoir la soufflerie. Par contre, à Namoungou dans l'est du Burkina Faso, les filles et sœurs des forgerons «s'érigeaient en aides pour le martelage et le soufflage ${ }^{27}$. Quant aux femmes des forgerons, elles sont potières.

Dans la forge, l'attention de l'apprenti est nécessaire pour assimiler les gestes nécessaires à la fabrication d'un objet. Pendant la formation, il est assis généralement à la droite du souffleur. Cependant les emplacements peuvent être adoptés par l'enfant selon sa convenance. Selon Boureima Nikiéma et Amidou Nikiéma, l'apprenant apprendra à ses dépens la position qui lui convient le mieux car entre la flamme du foyer, les objets chauds et le lieu du martelage, il occupera une place stratégique et adoptera finalement la position idéale à son apprentissage ${ }^{28}$. 
Les techniques d'apprentissage sont nombreuses. L'apprenant acquiert d'abord un savoir visuel. La technique consiste en l'observation de l'espace de la forge, de la disposition des différents éléments qui la composent, des gestes, des étapes de production des différents types d'objets et de l'organisation du travail.

Le savoir technique oral se transmet par des paroles peu nombreuses mais qui donnent les grandes directives pour la fabrication des objets. Elles font appel à l'écoute de celui qui apprend le langage du foyer.

L'éducation orale transmet les interdits et le savoir-faire technique. Il s'agit d'un langage qui se divulgue aussi à travers les gestes, les couleurs, les sons et les silences porteurs de messages. Un retrait d'objet du foyer, un arrêt brutal du martelage, un trempage brusque ou répété d'un objet sont porteurs de message. L'apprenant doit être en mesure de mémoriser tout ce qui se fait dans la forge dans le silence.

Le souffleur et le chef de la forge utilisent un langage maîtrisé par les initiés pour échanger. L'élève doit porter son attention sur ce qui se dit et observer en même temps les faits et gestes des maîtres. La répétition des paroles dans les mêmes circonstances permet à l'élève attentif de décoder les paroles. C'est le cas par exemple quand le forgeron chargé du martelage oublie de retirer l'objet qui subit le chauffage, le chef forgeron ne lui dit pas directement « l'objet brûle », il s'exprime en langage codé qui permet à ce dernier de comprendre sans que les visiteurs ne se rendent compte du problème. Il s'agit d'un langage connu des seuls initiés. Dans la forge, le foyer et l'enclume sont les maîtres de la parole. Le transfert des techniques se fait par la conjugaison de l'oralité, l'observation et la compréhension des sons, des couleurs et la pratique.

Par exemple, lorsque le forgeron récupère un morceau de loupe ou de fer de récupération, il le met au feu pour la transformation. Le métal est chauffé dans le foyer par l'actionnement des soufflets. La soufflerie est souvent assurée par les personnes un peu âgées ou les jeunes de moins de quinze ans. Comme chez les Bassars, « le souffleur profite par ailleurs de son intégration à l'équipe dans l'atelier pour observer le travail de près : c'est en somme un apprenti ${ }^{29}$. À partir de cet âge, il a des dispositions physiques lui permettant de participer au martelage. À ce sujet, Bruno Martinelli dit ceci : "L'apprentissage formel commence vers l'âge de quinze ans. À la forge l'apprenti passe par trois postes de travail qui correspondent à trois niveaux de formation ${ }^{30}$. Il actionne d'abord les soufflets, passe ensuite au martelage puis au travail et à la fabrication des outils ${ }^{31}$. À Sadaba, le souffleur atteint parfois l'âge de 15 ans à la soufflerie ${ }^{32}$. Après cet âge, il participe au martelage et peut accéder à la confection des objets. Le métal est extrait lorsqu'il est rouge vif pour être martelé sur l'enclume par des jeunes de bonne condition physique car cette étape de conception de l'objet nécessite de la force pour respecter les cadences de frappe. Le martelage permet de donner une forme à l'objet qui quitte l'état d'objet-pensée pour être un objet matériel. Il est successivement chauffé et martelé. Durant le martelage, le métal prend la couleur noire qui marque la fin de cette étape. L'objet est ensuite plongé dans l'eau pour être refroidit et solidifié. Cela accroît sa dureté, alors qu'un refroidissement à l'air libre contribuerait à le fragiliser et à le rendre cassant.

La répétition des gestes et des méthodes de martelage font ici appel à l'observation de l'apprenti, tout comme les différents aspects que prend l'objet que l'on fabrique. Quand la couleur du fer tend vers le blanc, il devient cassant pendant le martelage.

L'attention visuelle de l'apprenant est mise à contribution pour reconnaître les étapes de cuisson, de martelage, de chauffe et de bain de l'objet. La cadence des frappes et la force à engager dépendent de l'objet fabriqué. C'est au novice de suivre le travail qu'il possédera par la pratique et l'expérience. 
Pour ce qui est des sons, le forgeron indique que «la soufflerie parle». Le souffleur chante et communique à travers la soufflerie avec le forgeron. Les sons que dégage la soufflerie sont aussi porteurs de message. Les sonorités ou résonances sont différentes selon l'intensité de la flamme ou du martelage mais aussi selon le degré de cuisson du métal. On peut assimiler la soufflerie au bendré33. C'est en ce sens qu'à propos de la formation des jeunes, il est signalé que "They learn the craft by observing the elders, listening to sounds and assimilating the rules » 34 .

Dans la même vision, l'acquisition des techniques dans la forge utilise des procédés non compréhensibles par les concepts intellectuels européens actuels. Les techniques d'apprentissage sont propres à la société, voire à la communauté. L'élève fait appel ici à l'ouie et à la vue pour comprendre et détecter le message véhiculé par le foyer.

L'apprenant acquiert sa formation grâce à l'expérience qu'il accumule au fil du temps et à son esprit d'initiative. C'est pourquoi dans ses productions, il y a la réplique plus ou moins identique des objets faits par le maître, mais il y a ensuite les objets qui sont fruits de sa créativité. Il doit aussi savoir distinguer le «fer mou » du «fer dur » (acier) qui se côtoient dans le cas de la loupe mais aussi dans le fer de récupération ${ }^{35}$. En fonction de l'objet à fabriquer, son choix portera sur l'un ou l'autre type de fer. Autrefois, le koa ou loupe à «fer dur» permettait de produire des objets très résistants tandis que le koudmaasré (fer mou) servait aux autres types d'objets. La production du fer dur prenait plus de temps que celle du fer mou pour les objets à usage courant.

Toute cette formation se fait sous l'œil bienveillant des anciens qui observent et guident. Cette caractéristique a été notée chez les Fiko où "The elders, the old smiths are always present, observing and giving advice to the youngsters $» 36$.
Chez les Moose de Sadaba, l'apprenti reste lié à un maître forgeron, nul ne saurait apprendre tout seul. Pourtant ailleurs, on note la présence des apprentis mais aussi de ceux qui possèdent le « don de Dieu » et qui forgent sans passer par un maître. C'est en ce sens que Georges Celis indique que "Pour devenir forgeron, les informateurs interrogés affirment qu'il faut appartenir à une famille de forgerons. Ils distinguent entre celui qui a le «don de Dieu » et l'apprenti. Dans le premier cas il semble qu'il apprenne seul en imitant ce que font d'autres forgerons, tandis que dans l'autre cas, il invite un ancien à lui montrer comment on forge $»^{37}$. Dans le premier cas, lorsque l'aspirant se sent prêt, il invite les notables du village à une fête. Selon Georges Celis, ses compétences sont évaluées et les notables lui permettent de construire son hangar tandis que dans le second cas, c'est le maître qui invite les notables pour tester les capacités de son apprenti. Ses aptitudes évaluées, on lui permet également de construire sa forge s'il réussit avec succès la tâche que le jury composé des notables lui confie.

À Sadaba, la loupe a été entièrement remplacée par la matière première de récupération, même pour la majorité des objets de la forge tels l'enclume, les marteaux, les pinces. Les soufflets sont confectionnés non pas en argile mais sont de petits tonneaux de 10 litres, recouverts de peaux de chèvres auxquelles ils sont reliés par des élastiques issus de vielles chambres à air. À la place des cordes de lianes, le plastique est utilisé car il est abondant. Les tuyères sont des tuyaux en fer découpés dans de vieux cadres de vélos.

Le récipient servant à contenir l'eau de la trempe est parfois une boîte d'un kilogramme de tomate vide ou un plat en fer ou encore une poterie. Le charbon de bois est contenu dans une bassine en plastique d'une contenance de vingt litres. Dans la forge, le combustible est constitué de charbon de bois et de morceaux de bois. En général, les forgerons utilisent le premier type de combustible, mais lorsque l'énergie n'est pas suffisante pour la 
production d'un objet, le bois sert à accroître l'intensité.

\section{Conclusion}

L'activité de forge n'est plus ce qu'elle était autrefois à Sadaba. Les outils de travail sont en général issus du matériel industriel, et les productions sont aussi réalisées à partir du fer de récupération.

Les objets de la forge sont fortement concurrencés par les productions industrielles, mais gardent une place de choix auprès des paysans qui utilisent en majorité des lames de daba traditionnelles pour les cultures. Cependant le forgeron a perdu de son prestige et une partie de ses obligations techniques dont il n'a plus le monopole.

Les techniques d'apprentissage passent par la tradition orale et la pratique. L'apprentissage a aussi évolué car hors du travail de forge, des enfants de forgerons qui le désirent vont à l'école. Inscrits à l'école, ils participent sur leur temps libre aux travaux de forge. Ils allient de nos jours la formation scolaire à la formation à la forge.

1 DE BARROS Philip, The Bassar: Large Scale Iron Producers of the west African Savanna, Thèse de Doctorat, Los Angeles, University of California, 1985, $776 \mathrm{p}$.

$2 \mathrm{La}$ « smelting zone » correspond à la zone de réduction tandis que la « smithing zone » est la zone de forge.

3 DUGAST Stéphan « Entre four et forge ou jusqu'à quel point efficacité magique et savoir technique sont-ils conciliables ? (Bassar du Togo) ", dans ROBION-BRUNNER Caroline, MARTINELLI Bruno (éds.), Métallurgie du fer et sociétés africaines : bilans et nouveaux paradigmes dans la recherche anthropologique et archéologique, Oxford, Archaeopress, 2012, pp. 97-123.

4 CELIS Georges, « La métallurgie traditionnelle au Burundi, au Rwanda et au Buha, Essai de synthèse », Anthropos, 84, 1989 pp. 25-46.

5 Horé est un village de Guibaré. Kongoussi, chef-lieu de la province du Bam est situé au nord-ouest de ce village.
6 NIKIEMA Boureima, environ 100 ans, forgeron-métallurgiste, enquête réalisée dans le quartier de Piingin le 22 décembre 2013 à Sadaba.

7 NIKIEMA Boureima, environ 100 ans, forgeron-métallurgiste, enquête réalisée dans le quartier de Piingin le 22 décembre 2013 à Sadaba.

8 KIETHEGA Jean-Baptiste, La métallurgie lourde du fer au Burkina Faso, une technologie à l'époque précoloniale, Paris, Karthala, 2009, 500 p.

9 Le terme «pays Bisa » désigne la partie sud-est du Burkina Faso qui est occupée par l'ethnie des Bisa ou «Boussanssé ».

10 Les Benda désignent les griots chez les Moose. Ils sont les garants de l'histoire des sociétés et sont au service de la cour royale. Ils participent à toutes les cérémonies religieuses et coutumières.

${ }^{11}$ Les Fulbé sont les populations peules qui se retrouvent dans toutes les provinces du Burkina Faso.

12 Les Nakomsé désignent tous ceux qui se réclament descendants de Yennega, leur ancêtre. Ils exercent le pouvoir politique traditionnel dans le Moogo, pays des Moose.

13 Les Poéses sont des populations anciennement installées au Burkina Faso. Ils pratiquent la divination et on les retrouve surtout dans le centre du pays.

14 Les Setba sont des griots d'origine externe. Ils viennent du Mali ou du Niger. D'origine peule ou mandingue, ils participent aux cérémonies festives telles que les mariages et baptêmes. Ils récitent les louanges des personnes rencontrées et reçoivent des présents en contrepartie.

15 Les Yarsé désignent une ethnie du Burkina Faso. Ils viendraient du Mali actuel et se sont installés au Burkina Faso du XVIe au XVIIIe, voir au XIX ${ }^{e}$ siècle. Ce sont des commerçants qui pratiquent en majorité l'islam.

16 KIETHEGA, 2009, op. cit., p. 427.

17 NIKIEMA Boureima, environ 90 ans, enquête réalisée dans le quartier de Piingin à Sadaba le 22 décembre 2013.

18 KIETHEGA, 2009, op. cit., p. 426.

19 THIOMBIANO F. Elise, La production ancienne du fer dans le Gulmu: cas de Namoungou (Province du Gourma-Burkina Faso), Mémoire de Maîtrise, Ouagadougou, Université de Ouagadougou, 1990-1991, $150 \mathrm{p}$.

20 Le Pegba est la tuyère principale. Elle joue un rôle important dans la forge car elle est sacrée et reçoit de multiples offrandes en poulets.

21 On les appelle les pebsé qui désignent le pluriel de pebga. Ce sont des tuyères secondaires.

22 SOULIGNAC Raphaël et SERNEELS Vincent, « Forging with Dogon smiths (Mali) », dans DUNGWORTH David, DOONAN 
Roger (éds.), Accidental and Experimental archaeology, Londres, Historical Metallurgical Society, 2013, pp. 119-126.

23 CELIS, 1989, op. cit., p. 29.

24 NIKIEMA Salif, environ 95 ans, enquête réalisée dans le quartier de Tenkeingseega le 23 décembre 2013 à Sadaba.

25 MARTINELLI Bruno, "La mémoire en travail. À propos de la forge au Burkina Faso », CATEIS, 1, 1998, pp. 65-76.

26 SOULIGNAC et SERNEELS, 2013, op. cit., p. 121.

27 THIOMBIANO, 1990-1991, op. cit., p. 99.

28 NIKIEMA Boureima : 100 ans, Nikiéma Amidou, environ 80 ans, enquête réalisée dans le quartier de Piingin à Sadaba le 22 décembre 2013.

29 DUGAST Stéphan, "La pince et le soufflet, deux techniques de forge traditionnelle au Nord-Togo ", Journal des Africanistes, vol. $56 \mathrm{n}^{\circ} 2,1986$, pp. 29-53

30 MARTINELLI, 1998, op. cit., p. 67.

31 Ibid., p. 68.

32 NIKIEMA Salif : 95 ans, Nikièma Adama : 50 ans, habitants le quartier de Tenkeingseega à Sadaba. Enquête réalisée le 13 mai 2014.

33 NIKIEMA Salif : 95 ans, enquête réalisée le 23 décembre 2013 dans le quartier de Tenkeingseega à Sadaba. Dans son discours à l'Université de Laval, Joseph PARÉ, Président de l'Université de Ouagadougou, indique que "Pour Pacéré Titinga, il n'était pas possible de parler de la littérature africaine sous certaines de ses formes en usant de concepts européens. C'est dans cette optique qu'il a essayé de forger un nouveau terme mixte, composé de «bendré » signifiant en moré «le tam-tam parleur» et de « logie » (du grec «logos ») renvoyant à «étude » ou « science». La bendrologie est donc la science du tam-tam parleur. C'est cette science que maitre Pacéré a essayé de développer, parce qu'en dehors de l'écriture et de la parole, il existe dans la société africaine, en général, et chez les Mossé (ou Mossi) en particulier, d'autres moyens d'expression. ». "Manega, musée de la bendrologie, musée de la science du tam-tam parleur », Ici, c'est ailleurs. Consulté le 29/12/2013: allaroundzeworld.wordpress.com/.../manega-musee-de-la-

bendrologie/.

34 SOULIGNAC et SERNEELS, 2013, op. cit., p. 121.

35 NIKIEMA Salif : 95 ans et Nikiéma Adama 50 ans ; forgerons, enquête réalisée à Tenkeingseega le 23 décembre 2013 à Sadaba. 36 SOULIGNAC et SERNEELS, 2013, op. cit, p. 121.

37 CELIS Georges, "Fondeurs et forgerons ekonda, EquateurZaïre », Anthropos, 82, 1987, pp.109-134. 


\section{$\underline{\text { Sources orales }}$}

NIKIEMA Amidou : 80 ans, Nikiéma Boureima : 100 ans, Nikiéma Boukary, Nikiéma Wahabo ; forgerons à Sadaba, quartier de Piigin, enquête réalisée les 22 décembre 2013.

NIKIEMA Hamidou : environ 55 ans, Nikiéma Salif : 95 ans, Nikiéma Adama: environ 65 ans, Nikiéma Kader : 42 ans ; forgerons à Sadaba, quartier de Tenkeinseega, enquête réalisée les 23 décembre 2013 et le 13 mai 2014.

\section{Bibliographie}

CELIS Georges, « Fondeurs et forgerons ekonda, Equateur- Zaïre », Anthropos, 82, 1987, pp.109134.

CELIS Georges, "La métallurgie traditionnelle au Burundi, au Rwanda et au Buha, Essai de synthèse ", Anthropos, 84, 1989, pp. 25-46.

DE BARROS Philip, The Bassar: Large Scale Iron Producers of the west African Savanna, Thèse de Doctorat, Los Angeles, University of California, 1985, $776 \mathrm{p}$.

DUGAST Stéphan, «La pince et le soufflet, deux techniques de forge traditionnelle au Nord-Togo ", Journal des Africanistes, vol.56 $\mathrm{n}^{\circ} 2,1986$, pp. 2953 .

DUGAST Stéphan, «Entre four et forge ou jusqu'à quel point efficacité magique et savoir technique sont-ils conciliables? (Bassar du Togo)», dans Robion-Brunner Caroline, Martinelli Bruno (éds.), Métallurgie du fer et sociétés africaines : bilans et nouveaux paradigmes dans la recherche anthropologique et archéologique, Oxford, Archaeopress, 2012, pp. 97-123.
KIETHEGA Jean-Baptiste, La métallurgie lourde du fer au Burkina Faso, une technologie à l'époque précoloniale, Paris, Karthala, 2009, 500 p.

MARTINELLI Bruno, « La mémoire en travail. A propos de la forge au Burkina Faso », CATEIS, 1, 1998, pp. 65-76.

SOULIGNAC Raphaël et SERNEELS Vincent, « Forging with Dogon smiths (Mali)», in Dungworth David, Doonan Roger (éds.), Accidental and Experimental archaeology, London, Historical Metallurgical Society, 2013, pp. 119-126.

THIOMBIANO F. Elise, La production ancienne du fer dans le Gulmu: cas de Namoungou (Province du Gourma-Burkina Faso), Mémoire de Maîtrise, Ouagadougou, Université de Ouagadougou, 1990-1991, $150 \mathrm{p}$.

\section{SITES INTERNET}

«Manega, musée de la bendrologie, musée de la science du tam-tam parleur ", Ici, c'est ailleurs. Consulté le 29/12/2013 :

allaroundzeworld.wordpress.com/.../manegamusee-de-la-bendrologie/ 